\title{
Concise Asymmetric Synthesis and Pharmacological Characterization of All Stereoisomers of Glutamate Transporter Inhibitor TFB-TBOA and Synthesis of EAAT Photoaffinity Probes
}

Michele Leuenberger, ${ }^{\dagger}$, Andreas Ritler, ${ }^{\dagger}$ Alexandre Simonin, ${ }^{\ddagger},{ }^{\prime}$ Matthias A. Hediger ${ }^{\ddagger}, \S$ and Martin Lochner*,,$\uparrow$

†Department of Chemistry and Biochemistry, University of Bern, 3012 Bern, Switzerland.

Institute of Biochemistry and Molecular Medicine, University of Bern, 3012 Bern, Switzerland.

${ }^{\S}$ Swiss National Centre of Competence in Research, NCCR TransCure, Switzerland.

\begin{abstract}
Glutamate is the major excitatory neurotransmitter in the mammalian brain. Its rapid clearance after the release into the synaptic cleft is vital in order to avoid toxic effects and is ensured by several transmembrane transport proteins, so-called excitatory amino acid transporters (EAATs). Impairment of glutamate removal has been linked to several neurodegenerative diseases and EAATs have therefore received increased attention as therapeutic targets. $O$-benzylated L-threo- $\beta$-hydroxyaspartate derivatives have been developed previously as highly potent inhibitors of EAATs with TFB-TBOA ((2S,3S)-2-amino-3-((3-(4(trifluoromethyl)benzamido)benzyl)oxy)succinic acid) standing out as low-nanomolar inhibitor. We report the stereoselective synthesis of all four stereoisomers of TFB-TBOA in less than a fifth of synthetic steps than the published route. For the first time, the inhibitory activity and isoform selectivity of these TFBTBOA enantio- and diastereomers were assessed on human glutamate transporters EAAT1-3. Furthermore, we synthesized potent photoaffinity probes based on TFB-TBOA using our novel synthetic strategy.
\end{abstract}


Key Words: glutamate transporters; asymmetric synthesis; inhibitors; aspartate derivatives; isoform selectivity; photoaffinity probes.

\section{Introduction}

The involvement of glutamate in electrophysiological processes in the brain was first proposed and later confirmed in the 1950s. ${ }^{1}$ It has been established since that the glutamatergic system regulates most of the excitatory activities in the central nervous system and it is estimated that $80-90 \%$ of the neurons use glutamate as neurotransmitter. A crucial step is the rapid termination of glutamate excitation since a high concentration of glutamate in the synaptic cleft, as upon release from pre-synaptic vesicles, is leading to excitotoxicity over time, which in turn can result in neuronal damage. ${ }^{2}$

Rapid removal of extracellular glutamate is achieved by five selective transmembrane transport proteins belonging to the solute carrier (SLC) 1 family: EAAT1 (human gene SLC1A3, termed GLAST in rodents), EAAT2 (SLC1A2, GLT-1), EAAT3 (SLC1A1, EAAC1), EAAT4 (SLC1A6) and EAAT5 (SLC1A7). The first three are generally expressed in the brain and play a fundamental role in the termination of glutamate-mediated excitation at synapses. The latter two are very tissue specific and only found in Purkinje cells in the cerebellum (EAAT4) and in retina (EAAT5), respectively. EAAT3 is mainly located on the surface of post-synaptic neurons, whereas EAAT1 and EAAT2 are expressed in astrocytes. EAAT2 alone is responsible for the transport of $>90 \%$ of extracellular glutamate into astrocytes. Not surprisingly, impairment of the glial glutamate transporters in particular, is leading to excitotoxic effects and death of neurons. In fact, several studies have linked the reduced function or expression of glutamate transporters to neurodegenerative diseases such as amyotrophic lateral sclerosis (ALS), Alzheimer, Parkinson and Huntington disease. ${ }^{2,3}$ Thus, enhancing glutamate transport is a viable therapeutic strategy for neuroprotection and corresponding studies both in vitro and in vivo in a mouse model of ALS, for instance, 
have provided some encouraging results. ${ }^{4}$ Addressing glutamate transporters with modulators does not only pose a challenge in terms of isoform selectivity and dynamic expression levels; it has very recently been shown for GLT-1 at least, that they are highly mobile at the surface of astrocytes, which in turn shapes synaptic transmission. ${ }^{5,6}$

Several research groups have investigated the activity of small molecules, in particular L-glutamate (1) and L-aspartate (2) derivatives (Chart 1), to inhibit specific glutamate transporter isoforms. ${ }^{7}$

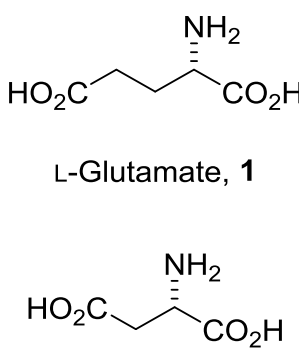

L-Aspartate, 2

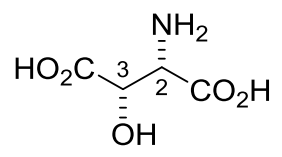

L-threo- $\beta$-Hydroxyaspartate, $(2 S, 3 S)-3$

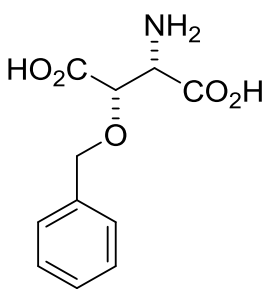

L-TBOA, $(2 S, 3 S)-4$

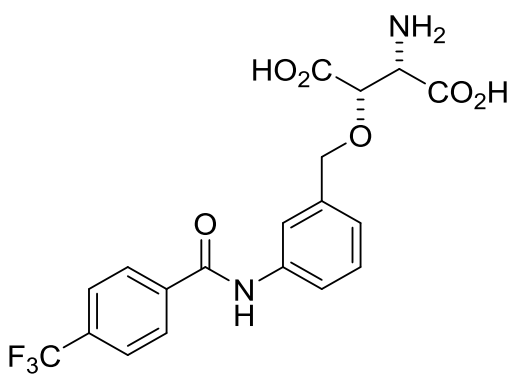

TFB-TBOA, $(2 S, 3 S)-5$

Chart 1. EAAT transporter substrates L-Glu and L-Asp and inhibitors 3-5 derived thereof.

Early on, threo-L- $\beta$-hydroxyaspartate $((2 S, 3 S)-3)$ was discovered to inhibit glutamate transport in synaptosomes and later identified as EAAC1 blocker. ${ }^{8}$ Shimamoto and co-workers developed a series of threo-L- $\beta$-hydroxyaspartate derivatives that are the most potent inhibitors of EAAT1-3 to date. From their first two studies, L-TBOA ((2S,3S)-4, L-threo- $\beta$-benzyloxyaspartate) emerged as the most active compound ( $\mathrm{IC}_{50}$ values in the low micromolar range). ${ }^{9-11}$ L-TBOA was used in numerous neurobiological studies and, among other things, co-crystallized with the homologous prokaryotic aspartate transporter $\mathrm{GltPh}$ from Pyroccocus hirokoshii (PDB ID: 2NWW). ${ }^{12}$ Notably, extending the structure at the phenyl ring, as exemplified by the best compound TFB-TBOA $((2 S, 3 S)-\mathbf{5})$, yielded EAAT2 and EAAT3 inhibitors with $\mathrm{IC}_{50}$ values in the low nanomolar range. ${ }^{13}$ 
Using the information from GltPh crystal structures ${ }^{12,14}$ other groups have used a structure-based screening approach to identify conformationally constrained analogues of aspartate and glutamate in a virtual compound library. ${ }^{15}$ This led to the discovery of a norbornane-type aspartate derivative which exhibited an $\mathrm{IC}_{50}$ of $1.4 \mu \mathrm{M}$ for EAAT2 and showed no inhibition of EAAT3. Moving away from amino acid scaffolds, Bunch and co-workers synthesized a library of substituted 5-oxo-5,6,7,8-tetrahydro-4Hchromenes and identified congeners that were potent inhibitors of EAAT1 ( $\mathrm{IC}_{50} 0.43 \mu \mathrm{M}$ for best compound) but displayed no noticeable inhibitory activity at EAAT2 and EAAT3. ${ }^{16-18}$

The published asymmetric syntheses of L-TBOA $((2 S, 3 S)-4)$ and TFB-TBOA $((2 S, 3 S)-\mathbf{5})$ are rather elaborate (10 and 21 synthetic steps, respectively). ${ }^{11,19}$ Our goal was to establish a general and concise synthetic procedure to prepare derivatives of TFB-TBOA that we envision to develop into molecular probes for studying glutamate transporters in cells. Here, we report the synthesis of all four stereoisomers of TFBTBOA (5) in only four synthetic steps and their inhibitory activity at human isoforms EAAT1-3. Furthermore, we used our novel synthetic strategy to generate two potent photoaffinity probes based on the TFB-TBOA scaffold.

\section{Results and Discussion}

\section{Chemistry}

In our retrosynthetic analysis the benzyl ether in $\mathbf{5}$ was disconnected which led to bromide $\mathbf{6}$ and the key intermediate $\beta$-hydroxyaspartate 3 (Scheme 1). After some literature research we envisaged to use asymmetric aminohydroxlyation developed by Sharpless and co-workers ${ }^{20-22}$ to install the two stereogenic centers in one reaction with high syn-selectivity to access the threo (2,3-syn) isomers (TBOA, Scheme 1, retrosynthetic path a). For the projected erythro (2,3-anti) isomers (EBOA, Scheme 1, retrosynthetic path b) we chose to utilize the Davis $\alpha$-hydroxylation methodology $y^{23}$ to diastereoselectively install the 
stereogenic centre at C-3. Thus, the projected synthetic plan would allow us to access all four stereoisomers of $\mathbf{5}$ in a very short four-step sequence starting either from fumarate $\mathbf{7}$ or protected aspartate $\mathbf{8}$.

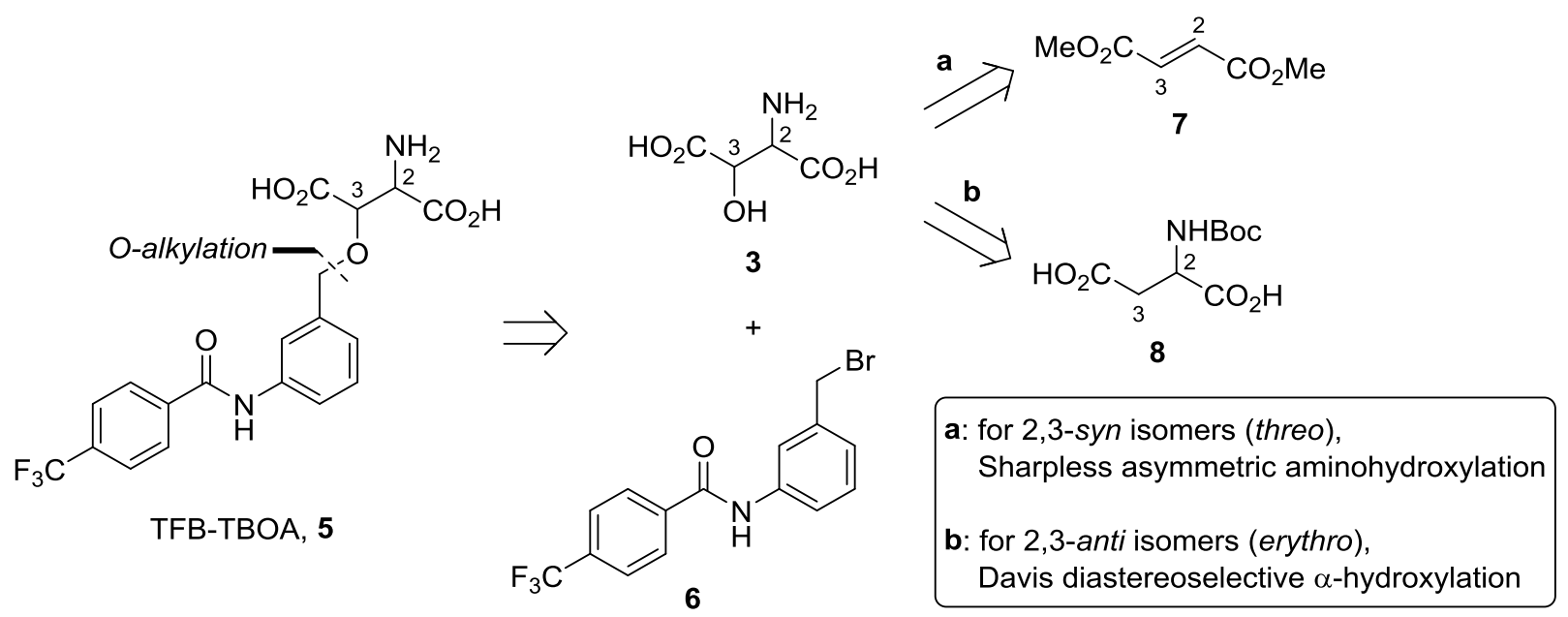

Scheme 1. Retrosynthetic analysis and synthetic strategy to access all four isomers of $\mathbf{5}$.

First, dimethyl fumarate (7) was converted to the tert-butyl diester 9 in order to streamline deprotection conditions at the end of the synthesis (Scheme 2). Following a recently published modification $^{24}$ of the Sharpless aminohydroxylation protocol yielded protected hydroxyaspartate $\mathbf{1 0}$ with complete diastereoselectivity (only syn isomers formed) in 65\%. The major improvement of this protocol is the use of 4-chlorobenzoyloxycarbamate (11) instead of the commonly used $N$-chlorocarbamate ${ }^{25}$ that has to be formed in situ from tert-butyl carbamate and freshly prepared tert-butyl hypochlorite under aqueous basic conditions. In the presence of chiral ligand (DHQD) $)_{2}$ PHAL the aminohydroxylation led to $(2 S, 3 S)-\mathbf{1 0}$ as major enantiomer, whereas using the pseudo-enantiomer (DHQ) ${ }_{2}$ PHAL as ligand gave mainly $(2 R, 3 R)-10$. In both cases the enantiomeric ratio (er) was determined to be $7: 3$ by chiral HPLC. It is important to run the aminohydroxylation reaction at $-15^{\circ} \mathrm{C}$ in order to suppress formation of 1,2-diol side products resulting from asymmetric dihydroxylation that is also operational under the reaction conditions. 
The free hydroxyl group of $\mathbf{1 0}$ was $O$-alkylated with bromide $\mathbf{6}^{26}$ (synthesis see Supporting Information). Global deprotection using TFA in chloroform led to enantioenriched isomers $(2 S, 3 S)-\mathbf{5}$ or $(2 R, 3 R)-\mathbf{5}$. By following the same synthetic route as shown in Scheme 2 but omitting the chiral phthalazine ligand in the asymmetric aminohydroxylation step we also synthesized the racemic mixture of the 2,3-syn (threo) isomers of $\mathbf{5}$ for biological assessment.

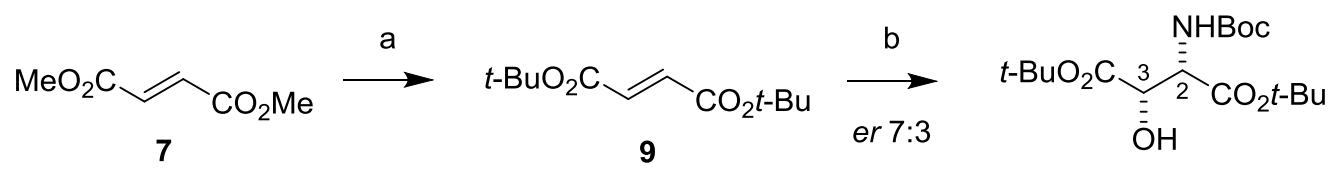

$(2 S, 3 S)-10$
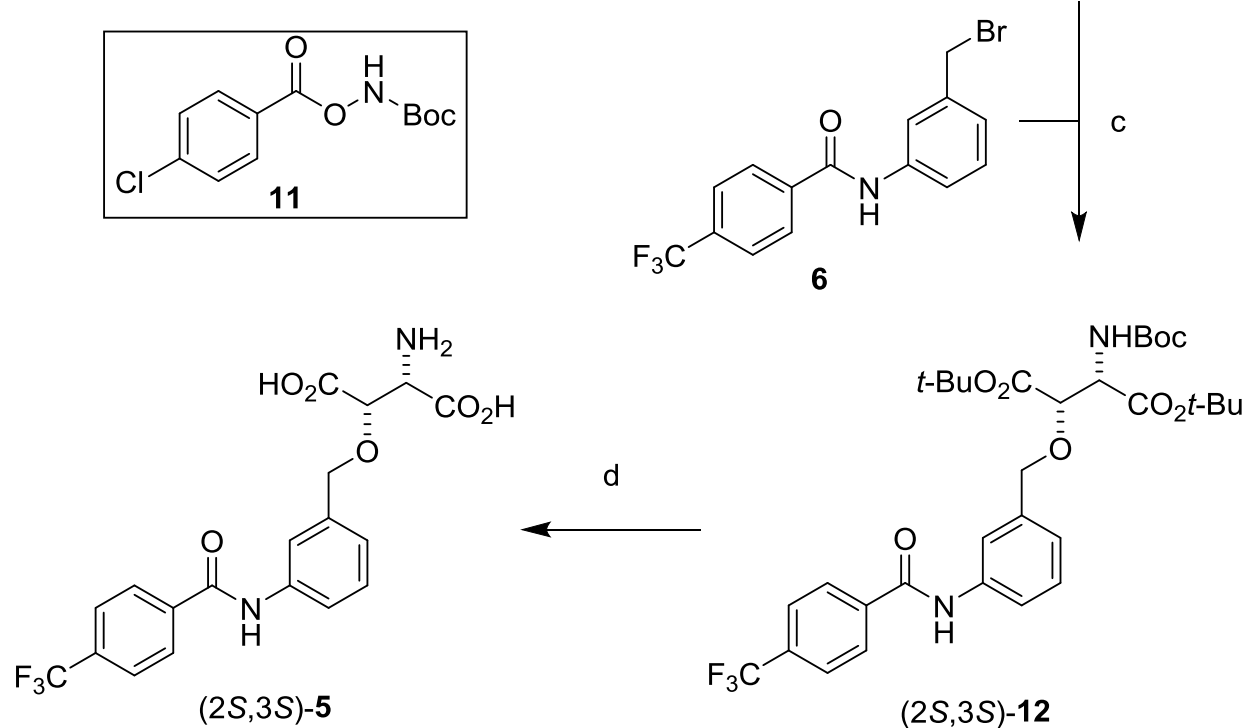

Scheme 2. Synthesis of 2,3-syn isomers (threo) of 5. Reagents and conditions: (a) $t$-BuOH, $n$-BuLi, THF, $0^{\circ} \mathrm{C}$; then $7,0^{\circ} \mathrm{C}$ to rt, $71 \%$. (b) for $(2 S, 3 S)-10$ (shown): $\mathrm{OsO}_{4}$, (DHQD) $)_{2} \mathrm{PHAL}, \mathbf{1 1}, \mathrm{H}_{2} \mathrm{O} / \mathrm{MeCN},-15^{\circ} \mathrm{C}$; then 9, $\mathrm{H}_{2} \mathrm{O} / \mathrm{MeCN},-15^{\circ}$ to $\mathrm{rt}, 65 \%$, er 70:30. For $(2 R, 3 R)-\mathbf{1 0}$ (not shown): $\mathrm{OsO}_{4},(\mathrm{DHQ})_{2} \mathrm{PHAL}, \mathbf{1 1}$, $\mathrm{H}_{2} \mathrm{O} / \mathrm{MeCN},-15^{\circ} \mathrm{C}$; then $9, \mathrm{H}_{2} \mathrm{O} / \mathrm{MeCN},-15^{\circ}$ to rt, $63 \%$, er 69:31. (c) $\mathrm{NaH}, \mathrm{DMF},-15^{\circ} \mathrm{C}$; then $6,-15^{\circ} \mathrm{C}$ to rt, $99 \%$ for $(2 S, 3 S)-\mathbf{1 2}, 98 \%$ for $(2 R, 3 R)-\mathbf{1 2}$. (d) TFA, $\mathrm{CHCl}_{3}, \mathrm{rt}, 84 \%$ for $(2 S, 3 S)-\mathbf{5}$, quant. for $(2 R, 3 R)-\mathbf{5}$. 
The synthesis of the two 2,3-anti (erythro) enantiomers started from enantiomerically pure $\mathrm{N}$-Boc protected L- or D-aspartate that was first transformed into the corresponding di-tert-butyl ester $\mathbf{1 3}$ (Scheme 3) in order to simplify deprotection at the end.
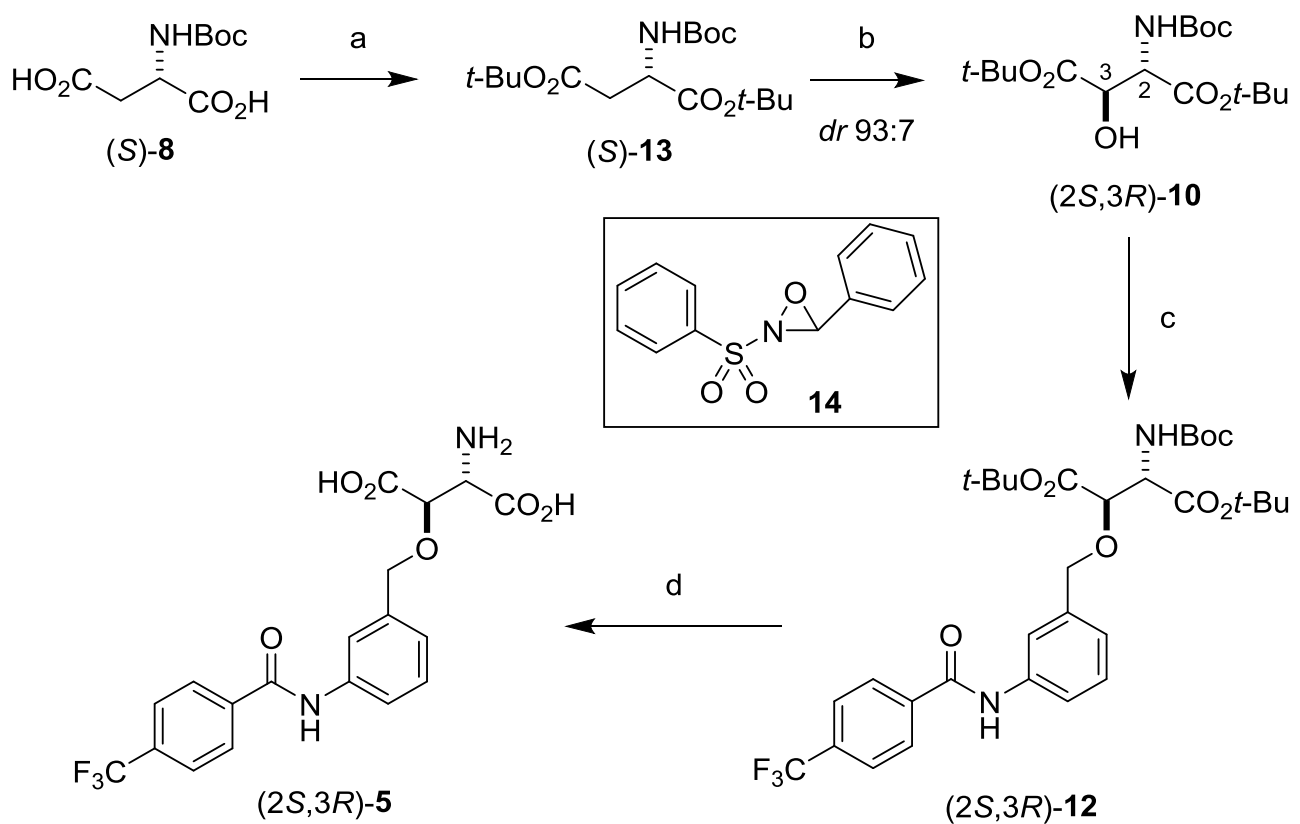

Scheme 3. Synthesis of 2,3-anti isomers (erythro) of 5. Reagents and conditions: (a) $\mathrm{CuCl}, \mathrm{DIPC}, \mathrm{t}-\mathrm{BuOH}$; then $\mathbf{8}, \mathrm{CH}_{2} \mathrm{Cl}_{2}$, reflux, $79 \%$ for $(S)-\mathbf{1 3}, 97 \%$ for $(R)-\mathbf{1 3}$. (b) NaHMDS, THF, $-78^{\circ} \mathrm{C}$; then $\mathbf{1 4}, \mathrm{THF},-78^{\circ} \mathrm{C}$, $22 \%$ and $d r 93: 7$ for $(2 S, 3 R)-\mathbf{1 0}, 26 \%$ and $d r 92: 8$ for $(2 R, 3 S)-\mathbf{1 0}$. (c) $\mathrm{NaH}, \mathbf{6}, \mathrm{DMF},-15^{\circ} \mathrm{C}$ to $0{ }^{\circ} \mathrm{C}, 19 \%$ for $(2 S, 3 R)-\mathbf{1 2}, 21 \%$ for $(2 R, 3 S)-\mathbf{1 2}$. (d) TFA, $\mathrm{CHCl}_{3}, \mathrm{rt}, 82 \%$ for $(2 S, 3 R)-\mathbf{5}, 96 \%$ for $(2 R, 3 S)-\mathbf{5}$.

Unexpectedly, this protection step failed or resulted in very low yields when employing commonly used conditions. A recently published protoco ${ }^{27}$ utilizing di-iso-propylcarbodiimide (DIPC), $t$-BuOH and $\mathrm{CuCl}$ as reagents ultimately worked best for us. The $\alpha$-hydroxylation reaction developed by Davis and co-workers is a powerful methodology to stereoselectively install hydroxyl groups next to carbonyl functions. ${ }^{28}$ In our particular case, treatment of fully protected aspartate $\mathbf{1 3}$ with NaHMDS at low temperature generated the 
corresponding ester enolate which was then almost exclusively attacked by the oxaziridine $\mathbf{1 4}$ from the opposite face of the $N$-Boc group, yielding the 2,3-anti hydroxylated product with high diastereoselectivity ( $d r$ 92:8-93:7). The isolated yield of the $\alpha$-hydroxylated products was low and a large amount of unreacted starting material was usually recovered. The $O$-alkylation of the 2,3-anti (erythro) alcohols $\mathbf{1 0}$ proved much more troublesome as we observed epimerization and elimination at reaction temperatures above $5^{\circ} \mathrm{C}$. Monitoring epimerization (by ${ }^{1} \mathrm{H}$ NMR) is advisable since the separation of diastereomers 2,3-anti-12 and 2,3-syn-12 is extremely difficult. Interestingly, once purified and isolated, the 2,3-anti isomers of $\mathbf{1 2}$ appear to be configurationally stable even at elevated temperature $\left(60^{\circ} \mathrm{C}\right.$ for $24 \mathrm{~h}$ in DMSO- $\left.d_{6}\right)$. Global deprotection to yield the final compounds $(2 S, 3 R)-\mathbf{5}$ and $(2 R, 3 S)-\mathbf{5}$ was achieved by treatment with TFA in chloroform. Notably, the 2,3-anti isomers did not epimerize under the strong acidic conditions. For the biological assessment we also synthesized the racemic mixture of the 2,3-anti isomers of 5 by starting from racemic $N$-Boc aspartate and following the synthetic route depicted in Scheme 3.

The synthetic route depicted in Scheme 2 is amenable to rapidly access TFB-TBOA analogues. For instance, by alkylating alcohol $(2 S, 3 S)$-10 with the appropriate building blocks we generated diazirine- and benzophenone-TFB-TBOA photoaffinity probes $\mathbf{1 5}$ and $\mathbf{1 6}$ (Chart 2), respectively (details of their synthesis see Supporting Information). Diazirine and benzophenone moieties appended to pharmacophores are commonly used for photo-crosslinking studies with proteins and can help to map the binding site of the pharmacophore. $^{29,30}$

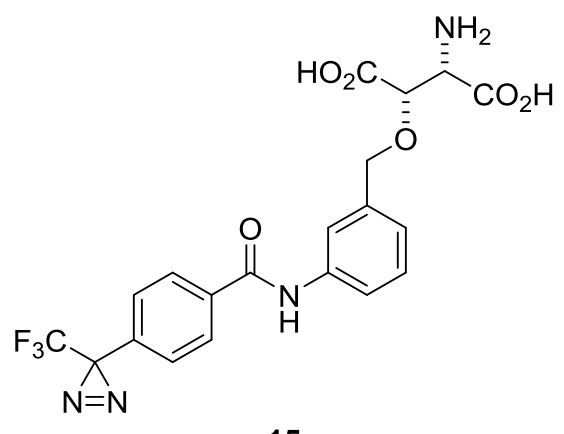

15

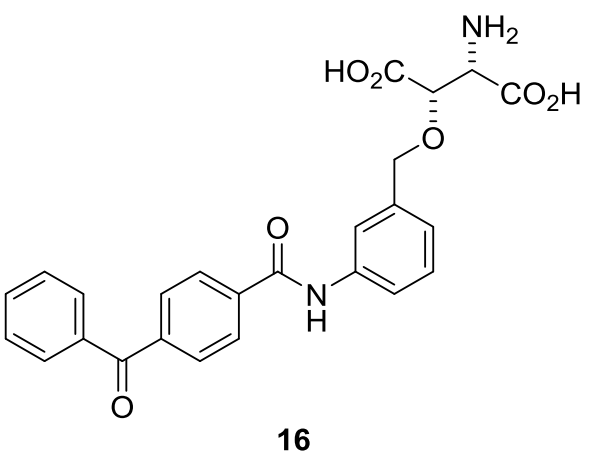

16

Chart 2. Structures of EAAT photoaffinity probes $\mathbf{1 5}$ and $\mathbf{1 6 .}$ 


\section{Biological Activity}

With all four stereoisomers of $\mathbf{5}$ in hand, we next examined their ability to inhibit glutamate uptake into cells. To this end, HEK293 cell lines stably expressing either human EAAT1, 2 or 3 were generated and transport of $\left[{ }^{3} \mathrm{H}\right]$-L-glutamate into these cells in the presence of $\mathbf{5}$ was determined using a similar assay set up as described previously (using $10 \mu \mathrm{M} \mathrm{L}$-glutamate in the transport assay). ${ }^{31}$ For all four stereoisomers of 5 we observed dose-dependent inhibition of $\left[{ }^{3} \mathrm{H}\right]$-L-glutamate transport (Figure 1).
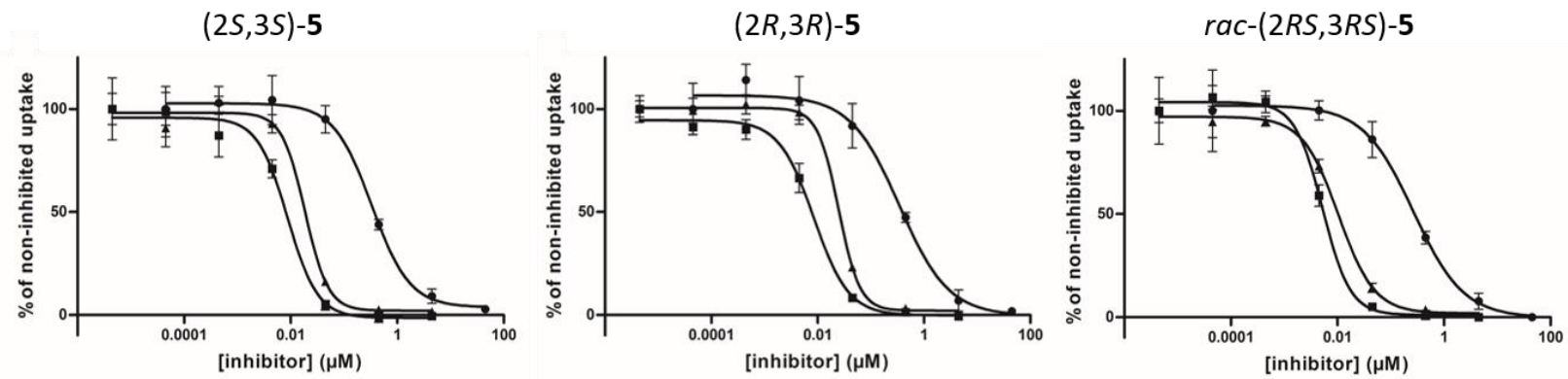

$\Delta$ EAAT1 - EAAT2 • EAAT3

$(2 S, 3 R)-5$

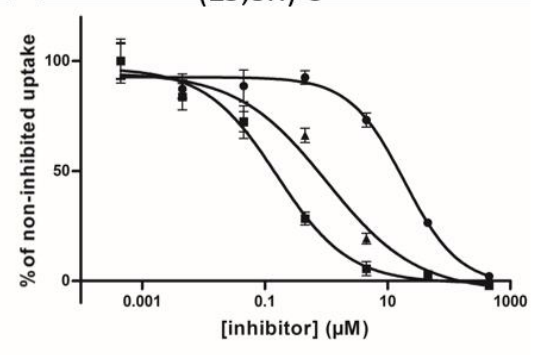

$(2 R, 3 S)-5$

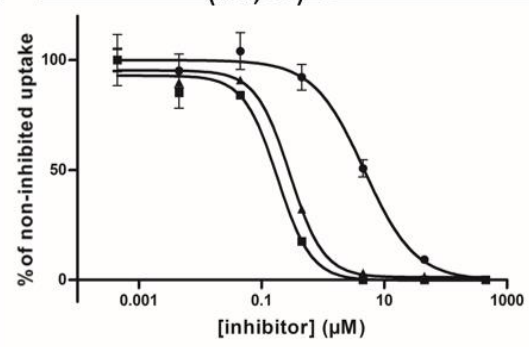

$\operatorname{rac}-(2 R S, 3 S R)-5$

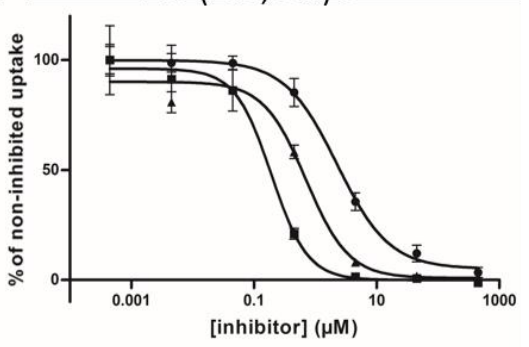

Figure 1. Dose-dependent inhibition of $\left[{ }^{3} \mathrm{H}\right]$-L-glutamate transport mediated by EAAT1 ( $\left.\mathbf{\Delta}\right)$, EAAT2 (匹) or EAAT3 (•), by stereoisomers of 5. Top panels: 2,3-syn isomers (threo, TFB-TBOA), bottom panels: 2,3-anti isomers (erythro, TFB-EBOA). Dose-inhibition curves shown are representative experiments. LGlutamate concentration used in the transport assay was $10 \mu \mathrm{M}$. All data are means of 3 measurements \pm SD. 
Overall, the 2,3-syn (threo, TFB-TBOA) isomers of $\mathbf{5}$ are more potent inhibitors of glutamate uptake facilitated by EAAT1, 2 or 3 than the 2,3-anti (erythro, TFB-EBOA) isomers by one to two orders of magnitude (Table). This is in line with what was observed for the stereoisomers of $\beta$-benzyloxyaspartate, where for instance L-TBOA $((2 S, 3 S)-4)$ was a more active inhibitor than L-EBOA $((2 S, 3 R)-4) .{ }^{11}$ It is interesting to note, however, that the difference in inhibitory potency of the $\beta$-benzyloxyaspartate stereoisomers 4 is more or less pronounced depending on the glutamate transporter isoform studied; an observation that we have also made with our synthesized stereoisomers of $\mathbf{5}$.

Table. Inhibiton of Human Glutamate Transporter-mediated $\left[{ }^{3} \mathrm{H}\right]-\mathrm{L}-$ Glutamate Uptake by TFB-TBOA and TFB-EBOA Stereoisomers. ${ }^{a}$

\begin{tabular}{l|lllc}
\hline \multicolumn{2}{c}{ compound } & \multicolumn{3}{c}{$\mathrm{IC}_{50}(\mathrm{nM})$} \\
\cline { 3 - 5 } & & EAAT1 & EAAT2 & EAAT3 \\
\hline \multirow{3}{*}{$2,3-$ syn, threo TFB-TBOA } & commercial $(S, S)$-TFB-TBOA & $8.2 \pm 3.6$ & $9.3 \pm 1.0$ & $248 \pm 76$ \\
& synthetic TFB-TBOA, $(2 S, 3 S)-5^{b}$ & $20.7 \pm 3.9$ & $9.9 \pm 1.3$ & $284 \pm 34$ \\
& $(2 R, 3 R)-5^{b}$ & $19.2 \pm 4.2$ & $12.2 \pm 4.1$ & $412 \pm 86$ \\
& rac-(2RS,3RS)-5 & $10.1 \pm 1.5$ & $6.4 \pm 1.8$ & $228 \pm 37$ \\
\hline \multirow{3}{*}{$2,3-$ anti, erythro TFB-EBOA } & $(2 S, 3 R)-5$ & $1051 \pm 97$ & $175 \pm 65$ & $13824 \pm 5007$ \\
& $(2 R, 3 S)-5$ & $335 \pm 96$ & $181 \pm 68$ & $2399 \pm 1690$ \\
& rac-(2RS,3SR)-5 & $619 \pm 73$ & $179 \pm 88$ & $5208 \pm 2830$ \\
\hline
\end{tabular}

${ }^{a} \mathrm{~L}-$ Glutamate concentration used in the transport assay was $10 \mu \mathrm{M}$. All data are means of at least 3 independent experiments performed in triplicate \pm SD.

${ }^{b}$ Enantioenriched, er 7:3 
In terms of isoform selectivity, EAAT2 was inhibited most strongly by all four stereoisomers of $\mathbf{5}$, followed by EAAT1 and then EAAT3, for the latter of which we obtained $\mathrm{IC}_{50}$ values in the high nanomolar to low micromolar range.

The $\mathrm{IC}_{50}$ values for our synthetic TFB-TBOA $((2 S, 3 S)-\mathbf{5})$, even though only enantioenriched, were almost identical for EAAT2 and EAAT3 and very similar for EAAT1 compared to the values we obtained with commercially available $(S, S)$-TFB-TBOA. Furthermore, the $\mathrm{IC}_{50}$ values obtained with our assay are very comparable to literature values for $(S, S)$-TFB-TBOA, where $\left[{ }^{14} \mathrm{C}\right]$-L-glutamate uptake into COS-1 cells transiently expressing EAAT1, 2 or 3 gave $\mathrm{IC}_{50}$ values of $22 \pm 0.4 \mathrm{nM}, 17 \pm 1 \mathrm{nM}$ and $300 \pm 45 \mathrm{nM}$, respectively. ${ }^{13}$ When comparing the enantiomers $(2 S, 3 S)-5$ and $(2 R, 3 R)-5$ (both enantioenriched to the same degree, $\operatorname{er~} 7: 3)$ it appears that $(2 S, 3 S)-\mathbf{5}$ is only twice as potent an inhibitor of EAAT3 as $(2 R, 3 R)-\mathbf{5}$, but has very similar inhibitory activity at EAAT1 and EAAT2 compared to its enantiomer. Interestingly, the racemic mixture (50:50) of both 2,3-syn (threo) enantiomers (rac-(2RS,3RS)-5) gave very similar $\mathrm{IC}_{50}$ values compared to commercially available $(S, S)$-TFB-TBOA. This lack of stereoselectivity in inhibitor binding by EAAT1 and EAAT2 might seem unusual but is not unprecedented for transporters. Poly-specific binding of enantiomeric cyclic peptide inhibitors was also observed in the case of the ABC transporter Pglycoprotein (P-gp) due to mainly hydrophobic and aromatic interactions between the inhibitor and the transporter. ${ }^{32}$

The stereoselectivity in terms of inhibitor activity is more pronounced for the 2,3-anti (erythro, TFBEBOA) isomers of $\mathbf{5}$, in particular in the case of EAAT3; the $(2 R, 3 S)$-isomer of $\mathbf{5}$ was about five times more active than its enantiomer $(2 S, 3 R)-5$. Furthermore, $\mathrm{IC}_{50}$ values for the racemic mixture of both TFB-EBOA enantiomers lay between values of the two individual enantiomers. As for the 2,3-syn (threo) isomers above, the stereoselection of EAAT1 and EAAT2 for the 2,3-syn (erythro) isomers was weakly manifested.

Both photoaffinity probes $\mathbf{1 5}$ and $\mathbf{1 6}$ inhibited transport of $\left[{ }^{3} \mathrm{H}\right]$-L-glutamate via EAAT1-3 in a dosedependent manner (Figure S1). Inhibition of EAAT1 was significantly stronger by $\mathbf{1 5}$ than $\mathbf{1 6}\left(\mathrm{IC}_{50} 0.11 \pm\right.$ $0.02 \mu \mathrm{M}$ and $1.23 \pm 0.29 \mu \mathrm{M}$, respectively). Similarly, diazirine $\mathbf{1 5}$ was the slightly more active inhibitor 
than 16 at EAAT2 $\left(\mathrm{IC}_{50} 0.40 \pm 0.03 \mu \mathrm{M}\right.$ and $0.82 \pm 0.08 \mu \mathrm{M}$, respectively), however, for EAAT3 this was the opposite $\left(15: \mathrm{IC}_{50} 4.03 \pm 0.80 \mu \mathrm{M} ; 16: \mathrm{IC}_{50} 2.11 \pm 0.58 \mu \mathrm{M}\right)$. Overall, both photoaffinity probes are more potent inhibitors than the standardly used DL-TBOA ( $\mathrm{IC}_{50}$ values $48.0 \pm 4.1,7.0 \pm 1.1$ and $8.0 \pm 1.9$ $\mu \mathrm{M}$ for EAAT1, EAAT2 and EAAT3, respectively). ${ }^{11}$

\section{Conclusions}

We have developed a short and concise synthesis for the preparation of all four stereoisomers of TFB-TBOA, a known and potent inhibitor of glutamate uptake by excitatory amino acid transporters EAAT1-3. Our only four-step synthetic route relies on the Sharpless asymmetric aminohydroxylation to access the 2,3-syn (threo) isomers, whilst we have employed the Davis asymmetric $\alpha$-hydroxylation to prepare the 2,3-anti (erythro) isomers of TFB-TBOA. For the first time all four stereoisomers have been tested on human glutamate transporters EAAT1-3 and our data show that the correct relative stereochemistry is crucial for high inhibitory activity, with the 2,3-syn (threo) isomers being clearly more active than the 2,3-anti (erythro) isomers. On the other hand, the absolute stereochemistry plays a minor role and enantioselectivity was effectively only observed for EAAT3. This observation implies that it is probably not necessary to use enantiomerically pure $(S, S)$-TFB-TBOA for biological studies but the racemic mixture, that is very easily synthesized following our developed protocol, is equally potent. Following our synthetic strategy, two TFB-TBOA-derived photoaffinity probes have been synthesized which exhibited sub-micromolar to micromolar inhibitory activity at EAAT1-3 and therefore could be useful molecular tools for photo-crosslinking studies. Efforts to develop further EAAT molecular probes with improved activities are underway in our laboratory and will be reported in due course. 


\section{Methods}

General Procedure for the Synthesis of 2,3-syn $\beta$-hydroxyaspartates $(2 S, 3 S)-10$ and $(2 R, 3 R)$ 10. $\mathrm{OsO}_{4}(5 \mu \mathrm{mol})$ was added as an aqueous stock solution $\left(61 \mu \mathrm{L}\right.$ of $2 \% \mathrm{OsO}_{4}$ in $\left.\mathrm{H}_{2} \mathrm{O}\right)$ to a stirred solution of either (DHQD) 2 PHAL (14.5 $\mu \mathrm{mol}$, for obtaining the $S, S$-isomer) or (DHQ)2PHAL (for obtaining the $R, R$-isomer) and 4-chlorobenzoyloxycarbamate 11 (43.8 $\mu \mathrm{mol}$, synthesis see Supporting Information) in $\mathrm{MeCN}(2.5 \mathrm{~mL})$. After stirring at room temperature for 12-25 min, the yellow-brown reaction mixture was cooled to $-15^{\circ} \mathrm{C}$ and a solution of fumarate 9 (43.8 mmol, synthesis see Supporting Information) in $\mathrm{MeCN} / \mathrm{H}_{2} \mathrm{O} 4: 1(2.5 \mathrm{~mL})$ was added slowly over a period of $15-45 \mathrm{~min}$ at $-15^{\circ} \mathrm{C}$. Upon addition, the color of the reaction mixture changed to dark green. After completed addition it was left to warm up to room temperature, whereupon the color changed again to pale yellow. Reaction control revealed no more product formation after $4 \mathrm{~h}$. The reaction was quenched by addition of sat. aqueous $\mathrm{K}_{2} \mathrm{~S}_{2} \mathrm{O}_{5}$ soln. (1 mL) which resulted in the precipitation of a fine brown powder. After addition of $\mathrm{H}_{2} \mathrm{O}(20 \mathrm{~mL})$ and $\mathrm{EtOAc}(20 \mathrm{~mL})$ the phases were separated and the aqueous layer was extracted with EtOAc $(3 \times 20 \mathrm{~mL})$. The combined organic layers were washed with sat. aqueous $\mathrm{NaHCO}_{3}$ soln. $(2 \times 15 \mathrm{~mL})$ and brine $(15 \mathrm{~mL})$, dried over $\mathrm{Na}_{2} \mathrm{SO}_{4}$, filtered and concentrated under reduced pressure to afford the crude product. This was purified by flash column chromatography (silica gel, EtOAc/hexane 1:9) and gave syn- $\beta$-hydroxyaspartate as single diastereomer. For spectral data of (2S,3S)-10 and (2R,3R)-10 see Supporting Information.

\section{General Procedure for the Synthesis of 2,3-anti $\beta$-hydroxyaspartates $(2 S, 3 R)-10$ and $(2 R, 3 S)$ -}

10. A solution of $N$-Boc protected $(S)$ - or $(R)$-aspartate diester $\mathbf{1 3}(0.87 \mathrm{mmol})$ in dry THF $(30 \mathrm{~mL})$ was cooled to $-78^{\circ} \mathrm{C}$ and a solution of NaHMDS in THF (1M, $\left.2.17 \mathrm{~mL}, 2.17 \mathrm{mmol}\right)$ was added dropwise. After stirring for $2 \mathrm{~h}$ at $-78^{\circ} \mathrm{C}$ a solution of oxazirine 14 (1.04 mmol, synthesis see Supporting Information) in dry THF $(6 \mathrm{~mL})$ was added at $-78^{\circ} \mathrm{C}$ to the reaction mixture. The progress of the reaction was monitored by TLC (silica gel, EtOAc/hexane 2:8) and was complete after 30-45 min. The reaction was quenched at $-78^{\circ} \mathrm{C}$ by addition of a solution of camphorsulfonic acid $(3.6 \mathrm{mmol})$ in $\mathrm{THF}(3.6 \mathrm{~mL})$ and further diluted with EtOAc (100 mL). The organic phase was washed with aqueous $\mathrm{HCl}(10 \%, 100 \mathrm{~mL})$, sat. aqueous 
$\mathrm{NaHCO}_{3}$ soln. $(100 \mathrm{~mL})$ and brine $(100 \mathrm{~mL})$, dried over $\mathrm{Na}_{2} \mathrm{SO}_{4}$, filtered and concentration under reduced pressure. The diastereomeric purity of the crude products (determined by ${ }^{1} \mathrm{H}$ NMR) was $d r$ 92:8-94:6. The crude was purified twice by flash column chromatography (silica gel, first column: $\mathrm{CH}_{2} \mathrm{Cl}_{2} / \mathrm{MeOH}$ 99:1, second column EtOAc/hexane 1:9) to afford anti- $\beta$-hydroxyaspartate as single diastereomer. For spectral data of $(2 S, 3 R)-\mathbf{1 0}$ and $(2 R, 3 S)$-10 see Supporting Information. 


\section{Associated Content}

\section{Supporting Information}

Synthesis procedures and spectral data for synthetic intermediates and final compounds, purity assessment of final compounds and details of biological procedures and pharmacological evaluation. This material is available free of charge via the Internet at http://pubs.acs.org.

\section{Author Information}

Corresponding Author

*M.L.: phone: +41 31631 3311; fax +41 31631 4272; E-mail martin.lochner@ dcb.unibe.ch

Author Contributions

Michele Leuenberger: developed novel synthetic route, synthesized and characterized intermediate and target compounds, contributed to writing of manuscript. Andreas Ritler: synthesized and characterized intermediate and target compounds. Alexandre Simonin: generated stable cell lines, performed pharmacological assays, data analysis and contributed to writing of manuscript. Matthias Hediger: supervised the pharmacological analysis. Martin Lochner: supervised the chemistry, designed experiments and wrote the manuscript.

\section{Funding Sources}

This study was supported by the Swiss National Science Foundation (SNSF professorship PP00P2_123536 and PP00P2_146321 to M.L., and through the National Center of Competence in Research (NCCR) TransCure) and the University of Bern. 
Notes

The authors declare no competing financial interest.

\title{
Acknowledgments
}

We thank the analytical services from the Department of Chemistry and Biochemistry, University of Bern, for measuring ${ }^{1} \mathrm{H}$ NMR, ${ }^{13} \mathrm{C}$ NMR and MS spectra.

\begin{abstract}
Abbreviations
ALS, amyotrophic lateral sclerosis; DIPC, N,N'-di-iso-propylcarbodiimide; DMAP, 4dimethylaminopyridine, EAAT, excitatory amino acid transporter, EBOA, erythro- $\beta$-benzyloxyaspartate, (2,3-anti)-2-amino-3-(benzyloxy)succinic acid; HMDS, hexamethyldisilazane; SLC, solute carrier (group of membrane transporter proteins); TBOA, threo- $\beta$-benzyloxyaspartate, (2,3-syn)-2-amino-3(benzyloxy)succinic acid; TFA, trifluoroacetic acid; TFB-TBOA, (2,3-syn)-2-amino-3-((3-(4(trifluoromethyl)benzamido)benzyl)oxy)succinic acid.
\end{abstract}




\section{References}

(1) Watkins, J. C., and Jane, D. E. (2006) The glutamate story. Br. J. Pharmacol. 147, S100-S108.

(2) Benarroch, E. E. (2010) Glutamate transporters: diversity, function, and involvement in neurological disease. Neurology 74, 259-264.

(3) Bunch, L., Erichsen, M. N., and Jensen, A. A. (2009) Excitatory amino acid transporters as potential drug targets. Expert Opin. Ther. Targets 13, 719-731.

(4) Rothstein, J. D., Patel, S., Regan, M. R., Haenggeli, C., Huang, Y. H., Bergles, D. E., Jin, L., Dykes Hoberg, M., Vidensky, S., Chung, D. S., Toan, S. V., Bruijn, L. I., Su, Z.-z., Gupta, P., and Fisher, P. B. (2005) $\beta$-Lactam antibiotics offer neuroprotection by increasing glutamate transporter expression. Nature 433, 73-77.

(5) Murphy-Royal, C., Dupuis, J. P., Varela, J. A., Panatier, A., Pinson, B., Baufreton, J., Groc, L., and Oliet, S. H. R. (2015) Surface diffusion of astrocytic glutamate transporters shapes synaptic transmission. Nat. Neurosci. 18, 219-226.

(6) Edwards, R. H. (2015) Mobile binding sites regulate glutamate clearance. Nat. Neurosci. 18, 166-168.

(7) Kanai, Y., Clémençon, B., Simonin, A., Leuenberger, M., Lochner, M., Weisstanner, M., and Hediger, M. A. (2013) The SLC1 high-affinity glutamate and neutral amino acid transporter family. Mol. Aspects Med. 34, 108-120.

(8) Kanai, Y., and Hediger, M. A. (1992) Primary structure and functional characterization of a high-affinity glutamate transporter. Nature 360, 467-471.

(9) Lebrun, B., Sakaitani, M., Shimamoto, K., Yasuda-Kamatani, Y., and Nakajima, T. (1997) New $\beta$ hydroxyaspartate derivatives are competitive blockers for the bovine glutamate/aspartate transporter. J. Biol. Chem. 272, 20336-20339. 
(10) Shimamoto, K., Lebrun, B., Yasuda-Kamatani, Y., Sakaitani, M., Shigeri, Y., Yumoto, N., and Nakajima, T. (1998) DL-threo- $\beta$-Benzyloxyaspartate, a potent blocker of excitatory amino acid transporters. Mol. Pharmacol. 53, 195-201.

(11) Shimamoto, K., Shigeri, Y., Yasuda-Kamatani, Y., Lebrun, B., Yumoto, N., and Nakajima, T. (2000) Syntheses of optically pure $\beta$-hydroxyaspartate derivatives as glutamate transporter blockers. Bioorg. Med. Chem. Lett. 10, 2407-2410.

(12) Boudker, O., Ryan, R. M., Yernool, D., Shimamoto, K., and Gouaux, E. (2007) Coupling substrate and ion binding to extracellular gate of a sodium-dependent aspartate transporter. Nature 445, 387 393.

(13) Shimamoto, K., Sakai, R., Takaoka, K., Yumoto, N., Nakajima, T., Amara, S. G., and Shigeri, Y. (2004) Characterization of novel L-threo- $\beta$-benzyloxyaspartate derivatives, potent blockers of the glutamate transporters. Mol. Pharmacol. 65, 1008-1015.

(14) Yernool, D., Boudker, O., Jin, Y., and Gouaux, E. (2004) Structure of a glutamate transporter homologue from Pyrococcus horikoshii. Nature 431, 811-818.

(15) Luethi, E., Nguyen, K. T., Bürzle, M., Blum, L. C., Suzuki, Y., Hediger, M., and Reymond, J.-L. (2010) Identification of selective norbornane-type aspartate analogue inhibitors of the glutamate transporter 1 (GLT-1) from the chemical universe generated database (GDB). J. Med. Chem. 53, 7236-7250.

(16) Jensen, A. A., Erichsen, M. N., Nielsen, C. W., Stensbøl, T. B., Kehler, J., and Bunch, L. (2009) Discovery of the first selective inhibitor of excitatory amino acid transporter subtype 1. J. Med. Chem. 52, 912-915.

(17) Erichsen, M. N., Huynh, T. H. V., Abrahamsen, B., Bastlund, J. F., Bundgaard, C., Monrad, O., Bekker-Jensen, A., Nielsen, C. W., Frydenvang, K., Jensen, A. A., and Bunch, L. (2010) 
Structure-activity relationship study of first selective inhibitor of excitatory amino acid transporter subtype 1: 2-amino-4-(4-methoxyphenyl)-7-(naphthalen-1-yl)-5-oxo-5,6,7,8-tetrahydro-4Hchromene-3-carbonitrile (UCPH-101). J. Med. Chem. 53, 7180-7191.

(18) Huynh, T. H. V., Shim, I., Bohr, H., Abrahamsen, B., Nielsen, B., Jensen, A. A., and Bunch, L. (2012) Structure-activity relationship study of selective excitatory amino acid transporter subtype 1 (EAAT1) inhibitor 2-amino-4-(4-methoxyphenyl)-7-(naphthalen-1-yl)-5-oxo-5,6,7,8-tetrahydro4H-chromene-3-carbonitrile (UCPH-101) and absolute configurational assignment using infrared and vibrational circular dichroism spectroscopy in combination with ab initio Hartree-Fock calculations. J. Med. Chem. 55, 5403-5412.

(19) Shimamoto, K. (2003) $\beta$-Benzyloxyaspartate derivatives with amino group on benzene ring. WO2003/000698 A1.

(20) Li, G., Chang, H.-T., and Sharpless, K. B. (1996) Catalytic asymmetric aminohydroxylation (AA) of olefins. Angew. Chem. Int. Ed. 35, 451-454.

(21) Bodkin, J. A., and McLeod, M. D. (2002) The Sharpless asymmetric aminohydroxylation. J. Chem. Soc., Perkin Trans. 1, 2733-2746.

(22) Herranz, E., Biller, S. A., and Sharpless, K. B. (1978) Osmium-catalyzed vicinal oxyamination of olefins by $N$-chloro- $N$-argentocarbamates. J. Am. Chem. Soc. 100, 3596-3598.

(23) Davis, F. A., Chattopadhyay, S., Towson, J. C., Lal, S., and Reddy, T. (1988) Chemistry of oxaziridines. 9. Synthesis of 2-sulfonyl- and 2-sulfamyloxaziridines using potassium peroxymonosulfate (oxone). J. Org. Chem. 53, 2087-2089.

(24) Harris, L., Mee, S. P. H., Furneaux, R. H., Gainsford, G. J., and Luxenburger, A. (2011) Alkyl 4chlorobenzoyloxycarbamates as highly effective nitrogen source reagents for the base-free, intermolecular aminohydroxylation reaction. J. Org. Chem. 76, 358-372. 
(25) Li, G., Angert, H. H., and Sharpless, K. B. (1996) N-Halocarbamate salts lead to more efficient catalytic asymmetric aminohydroxylation. Angew. Chem. Int. Ed. 35, 2813-2817.

(26) Pignataro, L., Bovio, C., Civera, M., Piarulli, U., and Gennari, C. (2012) A library approach to the development of BenzaPhos: Highly efficient chiral supramolecular ligands for asymmetric hydrogenation. Chem. Eur. J. 18, 10368-10381.

(27) Falck, J. R., Sangras, B., and Capdevila, J. H. (2007) Preparation of $N{ }^{t}{ }^{\mathrm{B} o c}$ L-glutathione dimethyl and di-tert-butyl esters: Versatile synthetic building blocks. Bioorg. Med. Chem. 15, 1062-1066.

(28) Hanessian, S., and Vanasse, B. (1993) Novel access to (3R)- and (3S)-3-hydroxy-L-aspartic acids, (4S)4-hydroxy-L-glutamic acid, and related amino acids. Can. J. Chem. 71, 1401-1406.

(29) Dubinsky, L., Krom, B. P., and Meijler, M. M. (2012) Diazirine based photoaffinity labeling. Bioorg. Med. Chem. 20, 554-570.

(30) Tanaka, Y., Bond, M. R., and Kohler, J. J. (2008) Photocrosslinkers illuminate interactions in living cells. Mol. Biosyst. 4, 473-480.

(31) Simonin, A., Montalbetti, N., Gyimesi, G., Pujol-Giménez, J., and Hediger, M. A. (2015) The hydroxyl side chain of a highly conserved serine residue is required for cation selectivity and substrate transport in the glial glutamate transporter GLT-1/SLC1A2. J. Biol. Chem. 290, 30464-30474.

(32) Aller, S. G., Yu, J., Ward, A., Weng, Y., Chittaboina, S., Zhuo, R., Harrell, P. M., Trinh, Y. T., Zhang, Q., Urbatsch, I. L., and Chang, G. (2009) Structure of P-glycoprotein reveals a molecular basis for poly-specific drug binding. Science 323, 1718-1722. 
Table of Contents Graphic

For Table of Contents Use Only

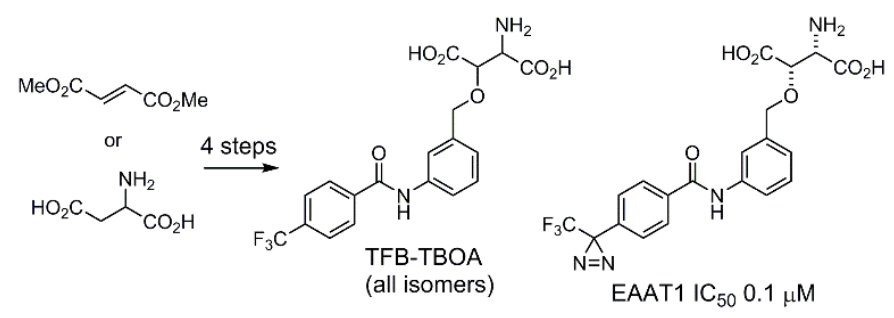

